\title{
La flore endémique du Nord-Est algérien face à la menace des espèces envahissantes
}

\author{
Nora Sakhraoui ${ }^{1}$, , Ratiba Boussouak ${ }^{1}$, Sophia Metallaoui ${ }^{1}$, Azzedine Chefrour ${ }^{2}$ et Azzedine Hadef ${ }^{1}$ \\ $1^{*}$ Département des Sciences de la Nature et de la Vie (SNV). Faculté des Sciences. Université 20 Aout 1955. BP 26 Route \\ d'El-Hadaiek. Skikda 21000 (Algérie). E-mail: sakhraouinora05@gmail.com \\ ${ }^{2}$ Département de Biologie. Faculté des Sciences de la Nature et de la Vie. Université Mohamed Cherif Messaadia. Souk \\ Ahras 41000 (Algérie).
}

\section{Correspondencia}

Nora Sakhraoui

e-mail: sakhraouinora05@gmail.com

Recibido: 11 abril 2019

Aceptado: 10 marzo 2020

Publicado on-line: 17 julio 2020

Editado por: Teresa Navarro

\begin{abstract}
Résumé
Nous avons établi pour la première fois dans la région de Skikda, les listes (liste actualisée et liste des taxons à rechercher) de la flore endémique et des espèces naturalisées jugées envahissantes en méditerranée, représentant une menace potentielle pour cette flore, en réalisant des prospections au niveau de douze communes. Cela a conduit à l'identification de 64 taxons endémiques dont plus de la moitié se trouve dans la liste actualisée (38 taxons). Ces plantes appartiennent à 26 familles, 46 genres et se répartissent en 50 espèces, 11 sousespèces et 3 variétés. Les endémiques algéro-tunisiennes sont les plus abondantes (30 taxons). 11 espèces envahissantes en méditerranée sont naturalisées dans notre région dont 5 colonisent des milieux naturels particulièrement riches en espèces endémiques. L'Acacia saligna, Carpobrotus edulis et Opuntia ficus-indica sont les principales espèces menaçantes de cette richesse biologique.
\end{abstract}

Mots clés : Listes floristiques, endémisme, chorologie, espèces envahissantes, Nord-Est algérien

\begin{abstract}
The endemic flora of Northeastern Algeria facing the threat of invasive plants. We established for the first time for the Skikda region, the lists (updated list and list of taxa to research) of endemic flora and naturalized species considered invasive in the Mediterranean, representing a potential threat to this flora, by conducting surveys at the level of twelve communes. This led to the identification of 64 endemic taxa more than half of which are in the updated list (38 taxa). These plants belong to 26 families, 46 genera and are divided into 50 species, 11 subspecies and 3 varieties. The Algerian-Tunisian endemics are the most abundant (30 taxa). 11 invasive species in the Mediterranean are naturalized in our region, 5 of which colonize natural environments particularly rich in endemic species. Acacia saligna, Carpobrotus edulis and Opuntia ficus-indica are the main threatening species of this biological richness
\end{abstract}

Key words: Floristic lists, endemism, chorology, invasive species, Northeastern Algeria.

\section{Introduction}

L'Algérie, au sein du bassin méditerranéen, est un pays à forte diversité biologique compte tenu de son paysage contrasté (Yahi et al. 2012). La région tellienne qui s'étend au-delà de $4 \%$ de la superficie du territoire national englobe à elle seule $2 / 3$ de la faune et de la flore algériennes. Le Tell littoral en particulier est considéré par plusieurs auteurs comme une zone d'intérêt majeur vu la richesse biologique et l'endémisme élevé qui le caractérisent (Quezel, 1964; Véla \& Benhouhou, 2007).

Faisant partie du Tell littoral, la wilaya de Skikda occupe une situation géographique particulière qui lui confère une position phytogéographique spécifique. En effet, si l'on se rapporte aux subdivisions biogéographiques retenues par Quezel \& Santa
(1962), Skikda se positionne dans trois secteurs. Son côté Est, principalement représenté par la plaine de Guerbes-Sanhadja, fait partie du secteur de la Numidie (K3), son côté Ouest, représenté essentiellement par le massif de Collo fait partie du secteur de la petite Kabylie (K2), tandis que son côté Sud est rattaché au secteur du Haut Tell constantinois (C1).

Les secteurs K2 et K3 ont été décrits comme des points chauds du bassin méditerranéen regroupant des espèces à grande valeur patrimoniale et possédant avec le secteur de la grande Kabylie (K1) l'indice de biodiversité le plus élevé à l'échelle nationale (Véla \& Benhouhou, 2007). Pour rendre possible sa conservation, cette richesse biologique doit être suffisamment connue pour chaque région du pays, la flore endémique, rare ou menacée restent 
prioritaires par rapport à toute tentative de préservation ou de valorisation. Or, les données relatives à la flore endémique sont fragmentées et les listes régionales ne sont pas établies pour les différentes wilayas algériennes.

Cette situation, nous a poussé à entreprendre ce travail dans la wilaya de Skikda (nord-est algérien), à travers lequel, nous avons essayé de rassembler toutes les observations relatives aux plantes endémiques rencontrées dans la région et d'établir, d'une part, une liste actualisée regroupant tous les taxons endémiques signalés dans les travaux récents et, d'autre part, une liste des taxons endémiques à rechercher, concernant toutes les plantes déjà observées dans la région mais absentes des travaux récents. Cette recherche bibliographique a été complétée par des études sur terrain et des observations personnelles faites lors des enquêtes effectuées dans certains points de la wilaya visant essentiellement à déterminer les menaces végétales pouvant nuire à cette richesse biologique.

\section{Matériel et méthodes}

\section{Zone d'étude}

La wilaya de Skikda est située au nord-est de l'Algérie. Elle est limitée au nord par la mer méditerranée, au sud-est par la wilaya de Guelma, au sud-ouest par les wilayas de Constantine et Mila, à l'est par la wilaya d'Annaba et à l'ouest par la wilaya de Jijel. Elle s'étend sur une superficie de $4140 \mathrm{Km}^{2}$, découpée administrativement en 13 daïras et 38 communes (Meghzili, 2015). En appliquant la méthode d'Emberger (1955) qui permet de caractériser le type du climat en méditerranée, la zone occidentale montagneuse représentée par le massif de Collo peut être classée dans l'étage bioclimatique humide (De Belair \& Sameraoui, 2000), l'étage subhumide couvre le reste de la wilaya (Abdi, 2015).

\section{Méthodes}

Selon la date d'observation, les taxons répertoriés ont été classés dans deux listes : Une liste actualisée et une liste des taxons à rechercher.

La liste actualisée des plantes endémiques a été élaborée selon deux méthodologies bien distinctes: (1) Une recherche bibliographique à travers laquelle tous les travaux publiés entre 2014 et 2018, traitant la flore du nord-est algérien ont été consultés, il s'agit de: Toubal et al. (2014), Hamel \& Meddad-Hamza (2016), Véla et al. (2016), Hamel et al. (2017a, 2017b), Boulemtafes et al. (2018) et Véla (2018). L'herbier de Gérard De Bélair, enregistré par l'Index Herbariorum sous le code ENSA, a également été consulté. Les taxons pris en considération sont ceux dont les observations ont été faites entre 2013 et 2017. (2) Des enquêtes sur terrain à travers la réalisation de 5 inventaires floristiques dans lesquels 88 relevés recueillis selon un échantillonnage aléatoire combiné à un échantillonnage systématique ont été réalisés essentiellement dans les zones de GuerbesSanhadja et Collo durant la période de 2014 à 2018 s'étendant du mois de février au mois de mai de chaque année (Fig. 1), représentant la principale période de floraison facilitant ainsi l'identification des espèces rencontrées. Les ouvrages utilisés pour l'identification des espèces sont Quezel \& Santa (1962, 1963) et Maire $(1958,1961,1987)$. Les spécimens récoltés ont été organisés dans des herbiers déposés au laboratoire de botanique du département des sciences de la nature et de la vie de I'Université de Skikda. Quant à la liste des plantes endémiques à rechercher sur le territoire de la wilaya de Skikda, elle a été élaborée à partir de Oberprieler (1998) pour le genre Anthemis, des anciennes flores notamment celles de Battandier \& Trabut (1888), Maire (1952), Quezel \& Santa (1963) et des herbiers. Les abréviations d'herbiers numériques nationaux et internationaux consultés sont présentées selon I'Index Herbariorum (Thiers, continuously updated): ENSA: Ecole Nationale Supérieure Agronomique; MPU: Université Montpellier II; P: Muséum National d'Histoire Naturelle.

Pour les deux listes, la nomenclature des espèces a été actualisée selon Dobignard \& Chatelain (20102013), quant à la chorologie, elle a été déterminée selon Quezel \& Santa (1962, 1963) puis actualisée, dans la mesure du possible, selon Pottier-Alapetite (1979, 1981), Fennane \& Ibn Tattou (1998), Oberprieler (1998), Le Floc'h et al. (2010), El Oualidi et al. (2012), IUCN (2013), Véla et al. (2016) et Véla (2018), les endémiques algériennes strictes ont été déterminées selon Dobignard \& Chatelain (20102013). Les familles botaniques sont représentées selon l'Angiosperm Phylogeny Group (APG IV, 2016). Les listes établies mettent également le point sur le degré de rareté déterminé selon Quezel \& Santa (1962, 1963), la synonymie et la répartition selon Dobignard \& Chatelain (2010-2013) et le type biologique selon Raunkiaer (1934).

Mise en évidence d'un nouveau type de menace : Au cours de la période s'étendant entre 2016 et 2018 , des enquêtes ont été menées dans 12 communes de la wilaya de Skikda à savoir : Ben Azouz, Bouchtata, Djendel, El Hadaiek, Filfilla, Hamadi Krouma, Mezedtchiche, Ramdane Djamel, Salah Bouchaour, Sidi Mezghich, Skikda et Tamalous, L'accessibilité des sites par voie routière était un critère majeur dans la sélection des zones prospectées. Les relevés floristiques effectués visaient à déterminer, d'un côté, les espèces naturalisées dans notre région jugées envahissantes en méditerranée, en s'appuyant sur les travaux de Brunel \& Tison (2005), Capdevilla Argüelles et al. (2006), Celesti-Grapow et al. (2009) et, d'un autre côté, la nature des milieux colonisés.

\section{Résultats}

\section{Richesse floristique des listes élaborées}

La méthodologie suivie nous a permis de dresser deux listes de référence de 64 plantes endémiques.

La liste actualisée comprend 38 taxons (Tab. 1) dont 12 ont été enregistrés dans les relevés floristiques réalisés sur terrain parmi 163 taxons 
inventoriés. 5 répertoriés dans la région de Collo: Genista numidica subsp numidica, G. tricuspidata, G. vepres, Quercus afares, Quercus canariensis et 7 dans la région de Guerbes-Sanhadja: Cyclamen africanum, Gagea algeriensis, Genista ferox, $G$. ulicina, Laurentia bicolor, Linaria pinifolia et Thymus numidicus. La recherche bibliographique a permis d'ajouter 26 taxons qui se répartissent en 19 espèces, 5 sous espèces et 2 variétés. La liste des taxons à rechercher comprend 26 taxons dont 12 taxons ont été signalés entre 1990-2011 et 14 taxons non jamais été revus depuis leur premier signalement dans la région (Tab. 2).

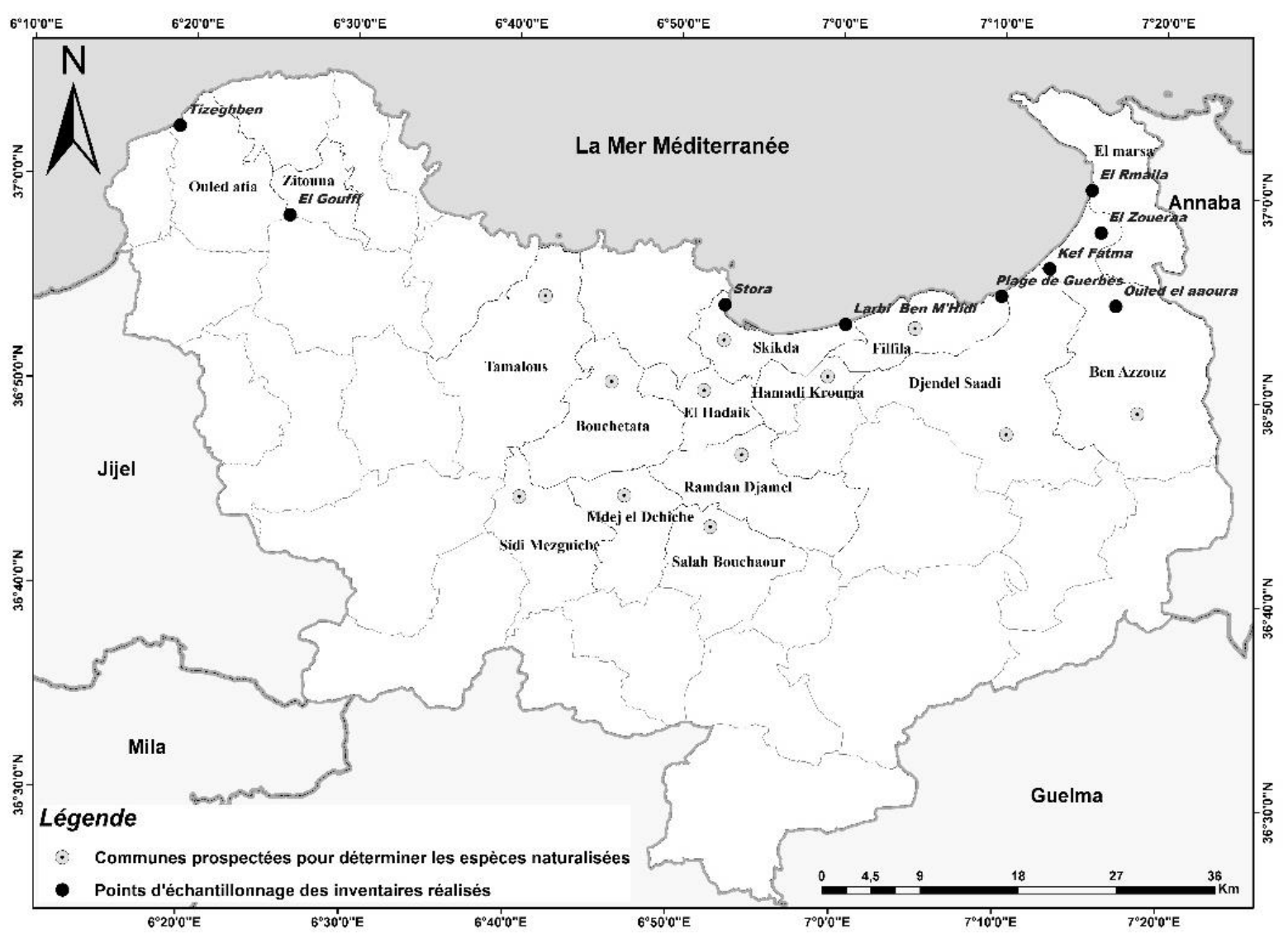

Figure 1. Localisation des points d'échantillonnage des inventaires réalisés et des communes prospectées.

Figure 1. Location of the sampling point inventories made and municipalities surveyed

\section{Spectre taxonomique}

Les taxons endémiques signalés dans les différentes stations de la wilaya, appartiennent essentiellement aux Eudicotylédones (50 taxons), les Monocotylédones sont représentées par 13 taxons, tandis que les Gymnospermes ne sont représentées que par un seul taxon.

Ces taxons se répartissent sur 26 familles (Fig. 2): La liste actualisée comprend 19 familles, la liste des taxons à rechercher comprend 15 familles. Les mieux représentées sont les Caryophyllaceae (7 taxons), Asparagaceae (7 taxons), Asteraceae (6 taxons), Fabaceae (6 taxons), Apiaceae (5 taxons), Brassicaceae (4 taxons), Campanulaceae (3 taxons), Lamiaceae (3 taxons), Crassulaceae, Fagaceae, Plumbaginaceae, Primulaceae et Scrophulariaceae (2 taxons chacune). Un total de 46 genres a été recensé: la liste actualisée comprend 29 genres, la liste des taxons à rechercher comprend 21 genres. Les mieux représentés sont Genista (5 taxons), Silene (4 taxons), Anthemis (3 taxons), Daucus (3 taxons) et Scilla (3 taxons).
Les taxons enregistrés appartiennent à 50 espèces, 11 sous-espèces et 3 variétés: 30 espèces, 6 sous-espèces et 2 variétés sont retrouvées dans la liste actualisée, tandis que 20 espèces, 5 sousespèces et 1 variété sont à rechercher.

\section{Distribution biogéographique des statuts d'endémisme}

Quatre statuts d'endémisme ont été enregistrés (Fig. 3). Les endémiques algériennes strictes sont représentées par 18 taxons $(28,12 \%): 8$ taxons sont à rechercher sur le territoire de la wilaya parmi lesquels 5 taxons $(62,5 \%)$ sont exclusivement localisés dans la région de Collo. $54 \%$ des sousespèces endémiques possèdent ce statut. Les endémiques algéro-tunisiennes sont représentées par 30 taxons et sont de ce fait les plus abondantes (46, 87\%). 9 taxons sont retrouvés dans la liste des taxons à rechercher. $50 \%$ des espèces endémiques appartiennent à ce statut.

Les endémiques algéro-marocaines sont les moins représentées dans la région avec seulement 3 taxons et un pourcentage ne dépassant pas $4,68 \%$. 


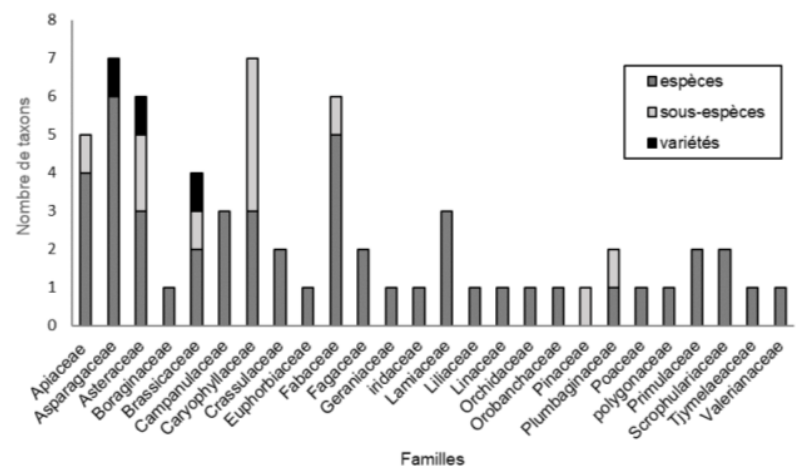

Figure 2. Nombres d'espèces, sous-espèces et variétés endémiques par famille.

Figure 2. Number of endemic species, subspecies and varieties per family

Les endémiques nord-africaines sont représentées par 13 taxons dont plus de 60\% est partagé entre l'Algérie, le Maroc et la Tunisie (Fig. 4). La chorologie de 35 plantes a changé: 9 taxons non signalés chez Quezel \& Santa (1962-1963) sont devenus endémiques algériens, 19 taxons sont devenus endémiques algéro-tunisiens et 5 taxons endémiques nord-africains après avoir été classés dans différentes catégories.

Au total, 17 taxons sont considérés comme rares sur le territoire national, 9 taxons assez rares et 5 taxons très rares représentant ainsi $48,31 \%$ de la flore endémique répertoriée. 10 endémiques algériennes strictes $(55,55 \%)$ sont classées dans ces trois niveaux de rareté (Fig. 5), dont 6 sont retrouvées dans la liste des taxons à rechercher. Les endémiques algéro-tunisiennes pouvant être classées dans les mêmes niveaux de rareté sont au nombre de 14 taxons $(46,66 \%)$ dont 11 sont retrouvés dans la liste actualisée.

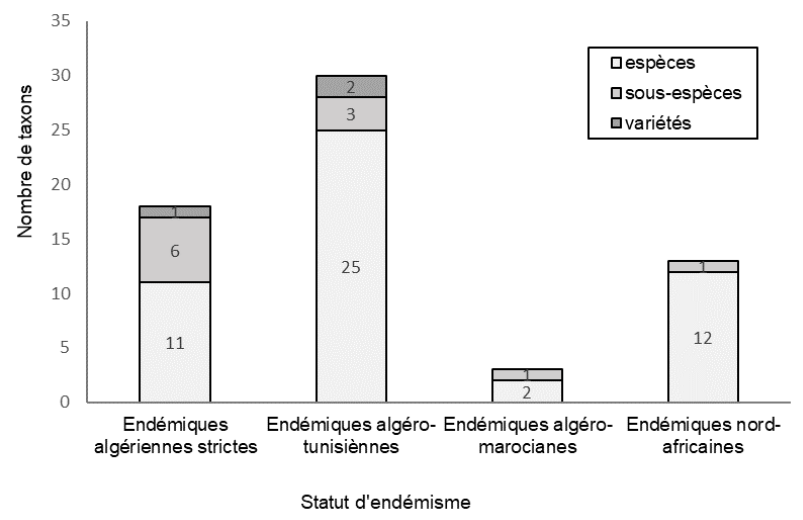

Figure 3. Nombre d'espèces, sous-espèces et variétés par statut d'endémisme.

Figure 3. Number of endemic species, subspecies and varieties by endemism status.

\section{Spectre biologique}

La flore endémique de la wilaya de Skikda appartient essentiellement aux hémicryptophytes (32, $81 \%)$, ces dernières sont de Quercus afares et $Q$. x numidica les plus abondantes dans la liste actualisée (13 taxons) tandis que les thérophytes sont dominantes dans la liste des taxons à rechercher (11 taxons).

\section{Mise en évidence de la menace des espèces envahissantes}

Nos prospections ont permis de détecter 11 espèces naturalisées jugées envahissantes dans les pays méditerranéens selon les travaux consultés, il s'agit d'Acacia saligna, Ailanthus altissima, Arundo donax, Austrocylindropuntia subulata, Carpobrotus edulis, Nicotiana glauca, Opuntia ficus-indica, Opuntia stricta, Oxalis pes-capreae, Ricinus communis et Tropaeolum majus.

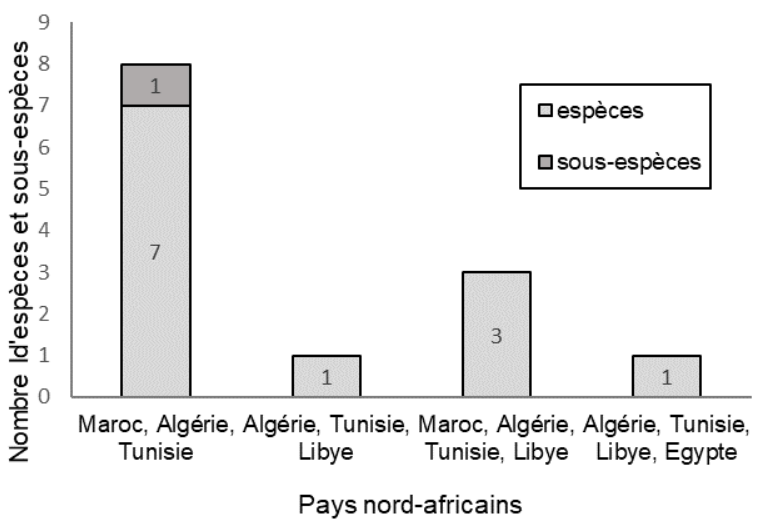

Figure 4. Répartition des endémiques nord-africaines.

Figure 4. Distribution of north-african endemic species.

Les communes côtières sont concernées par la naturalisation de toutes ces plantes introduites, les communes internes sont concernées par la naturalisation de l'Ailanthus altissima, Arundo donax, Opuntia ficus-indica, Oxalis pes-capreae et Ricinus communis. Les milieux colonisés sont principalement anthropiques (Tab. 3) mais la présence d'importantes populations de certaines espèces introduites en milieux naturels a été enregistrée dans plusieurs régions de la wilaya. L'Acacia saligna et Opuntia ficus-indica ont été retrouvées dans les maquis clairs et arborés, Carpobrotus edulis dans les plages et les dunes littorales, Arundo donax et Ailanthus altissima aux bords des oueds.

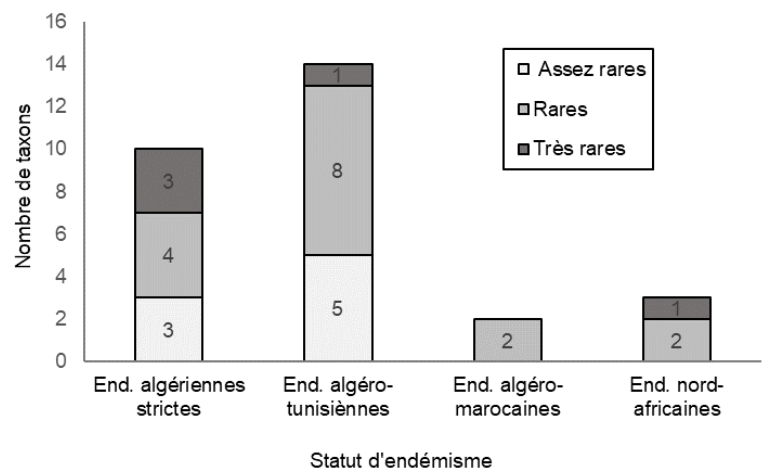

Figure 5. Répartition des taxons assez rares, rares et très rares selon le statut d'endémisme.

Figure 5. Distribution of quite rare, rare and very rare taxa according to the status of endemism. 


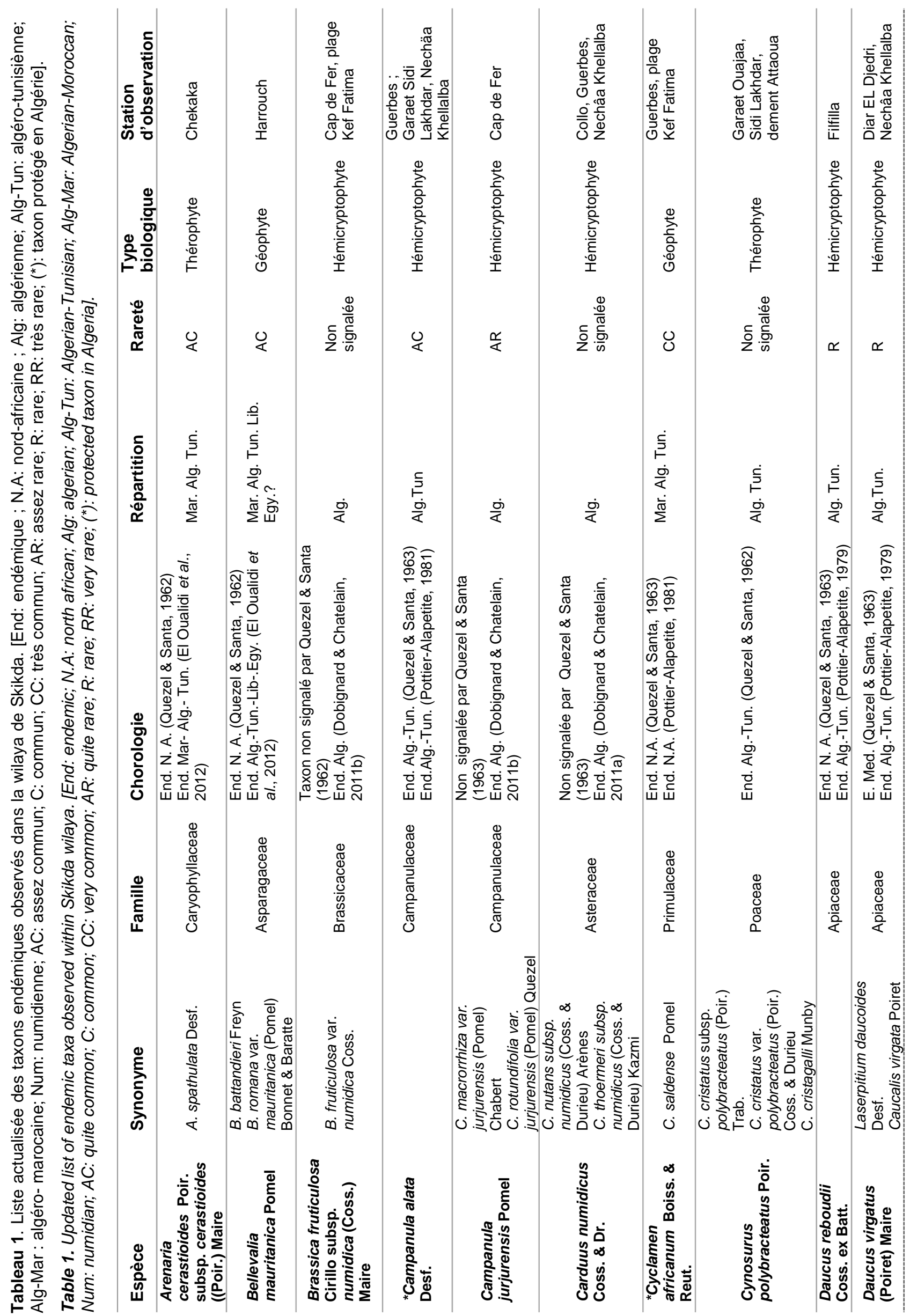




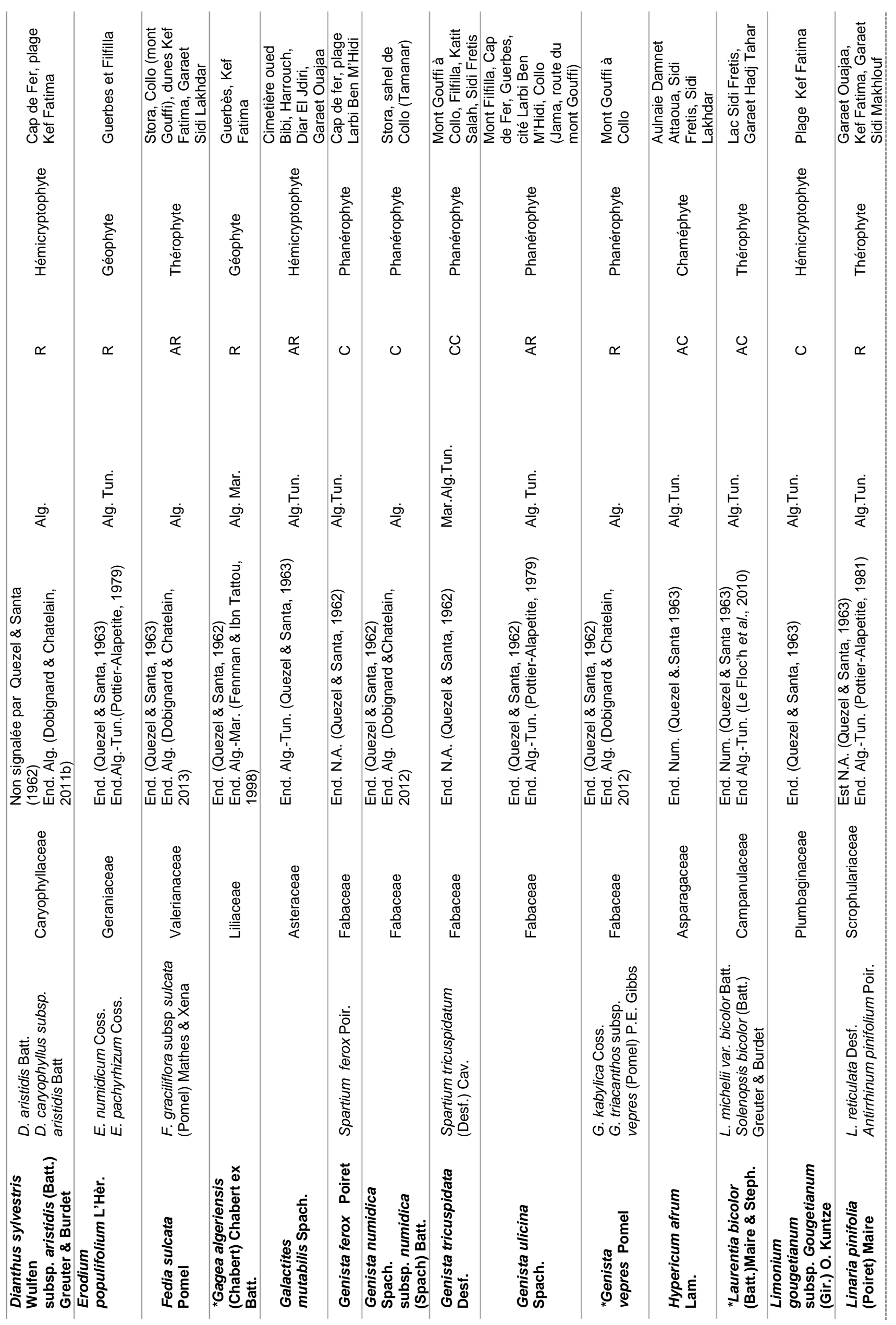







Tableau 2. Taxons endémiques déjà vus sur le territoire de la wilaya de Skikda mais absents des listes floristiques récentes (à rechercher). [End: endémique; N.A: nord-africaine; Alg: algérienne; Alg-Tun: algéro-tunisiènne; Alg-Mar: algéromarocaine; Num: numidienne; AC: assez commun; C: commun ; CC: très commun; AR: assez rare; R: rare; RR: très rare; $\left({ }^{*}\right)$ : taxon protégé en Algérie].

Table 2. Endemic taxa already seen within Skikda wilaya but absent from recent floristic lists (to research). [End: endemic; N.A: north african; Alg: algerian; Alg-Tun: Algerian-Tunisian; Alg-Mar: Algerian-Moroccan; Num: numidia; AC: quite common; $C$ : common; CC: very common; AR: quite rar ; $R$ : rare; $R R$ : very rare; $\left(^{*}\right)$ : protected taxon in Algeria

Taxons signalés entre 1990 -2011 (Herbier ENSA)

\begin{tabular}{|c|c|c|c|c|c|c|c|}
\hline Taxons & Synonyme & Famille & Chorologie & Répartition & Rareté & $\begin{array}{l}\text { Type } \\
\text { biologique }\end{array}$ & $\begin{array}{l}\text { Station } \\
\text { d'observation }\end{array}$ \\
\hline $\begin{array}{l}\text { Bellis prostrata } \\
\text { Pomel }\end{array}$ & $\begin{array}{l}\text { B. repens Lamk. } \\
\text { B. radicans Coss. } \\
\& \text { Dur. }\end{array}$ & Asteraceae & $\begin{array}{l}\text { Non signalée par Quezel } \\
\& \text { Santa (1963) } \\
\text { End. Alg.-Tun. (Pottier- } \\
\text { Alapetite, 1981) }\end{array}$ & $\begin{array}{l}\text { Mar. Alg. } \\
\text { Tun. }\end{array}$ & $\mathrm{RR}$ & Thérophyte & Garaet Bouina \\
\hline $\begin{array}{l}\text { Brassica } \\
\text { procumbens } \\
\text { (Poiret) Schultz. }\end{array}$ & $\begin{array}{l}\text { Sinapis } \\
\text { procumbens Poir. }\end{array}$ & Brassicaceae & $\begin{array}{l}\text { End. Num. (Quezel \& } \\
\text { Santa, 1962) }\end{array}$ & Alg.Tun. & C & Thérophyte & Cap de Fer \\
\hline $\begin{array}{l}\text { Borago longifolia } \\
\text { Poiret }\end{array}$ & & Boraginaceae & $\begin{array}{l}\text { End. N.A (Quezel \& Santa } \\
\text { 1963) } \\
\text { End. Alg.-Tun. (Pottier- } \\
\text { Alapetite, 1981) }\end{array}$ & Alg.Tun. & AR & $\begin{array}{l}\text { Hémicryptop } \\
\text { hyte }\end{array}$ & $\begin{array}{l}\text { Garaet Sidi } \\
\text { Lakhdar }\end{array}$ \\
\hline $\begin{array}{l}\text { Bunium fontanesii } \\
\text { (Pers.) Maire }\end{array}$ & $\begin{array}{l}\text { B. mauritanicum } \\
\text { Batt. }\end{array}$ & Apiaceae & $\begin{array}{l}\text { End. N. A. (Quezel \& } \\
\text { Santa, 1963) }\end{array}$ & $\begin{array}{l}\text { Mar. } \\
\text { Alg.Tun. } \\
\text { Lib. }\end{array}$ & $\mathrm{R}$ & Géophyte & Filfilla \\
\hline $\begin{array}{l}\text { Daucus gracilis } \\
\text { Steinh. }\end{array}$ & & Apiaceae & $\begin{array}{l}\text { Non signalée par Quezel } \\
\& \text { Santa (1963) } \\
\text { End. Alg. (Dobignard \& } \\
\text { Chatelain, 2011a) }\end{array}$ & Alg. & AR & Thérophyte & $\begin{array}{l}\text { Cap de Fer, } \\
\text { Filfilla (pont } \\
\text { borne) }\end{array}$ \\
\hline $\begin{array}{l}{ }^{*} \text { Maresia } \\
\text { malcolmioides } \\
\text { Coss. \& Dur. }\end{array}$ & $\begin{array}{l}\text { Malcolmia } \\
\text { malcolmioides } \\
\text { (Coss. \& Durieu) } \\
\text { Greuter \& Burdet } \\
\text { Sisymbrium } \\
\text { malcolmioides } \\
\text { Coss. \& Durieu }\end{array}$ & Brassicaceae & $\begin{array}{l}\text { End. (Quezel \& Santa, } \\
\text { 1962) } \\
\text { End.Alg.-Tun.(Pottier- } \\
\text { Alapetite, 1979) }\end{array}$ & Alg.Tun. & $\mathrm{RR}$ & Thérophyte & $\begin{array}{l}\text { Plage Kef } \\
\text { Fatima et Sidi } \\
\text { Fretis }\end{array}$ \\
\hline $\begin{array}{l}\text { Romulea numidica } \\
\text { Jord \& Fourr. }\end{array}$ & & Iridaceae & $\begin{array}{l}\text { End. Alg.-Mar. (Quezel \& } \\
\text { Santa, 1962) } \\
\text { End.Alg. - Mar. (El Oualidi } \\
\text { et al., 2012) }\end{array}$ & Mar. Alg. & $\mathrm{R}$ & Géophyte & Harrouch \\
\hline $\begin{array}{l}\text { Scilla numidica } \\
\text { Poir. }\end{array}$ & $\begin{array}{l}\text { Barnardia } \\
\text { numidica (Poir.) } \\
\text { Speta }\end{array}$ & Asparagaceae & $\begin{array}{l}\text { End. Alg.-Tun. (Quezel \& } \\
\text { Santa, 1962) } \\
\text { End.Alg.-Tun.-Lib. (El } \\
\text { Oualidi et al., 2012) }\end{array}$ & $\begin{array}{l}\text { Alg. Tun. } \\
\text { Lib. }\end{array}$ & C & Géophyte & Filfilla \\
\hline $\begin{array}{l}\text { Sedum pubescens } \\
\text { Vahl. }\end{array}$ & S. hispidum Desf. & Crassulaceae & $\begin{array}{l}\text { End. Alg.-Tun. (Quezel \& } \\
\text { Santa, 1962) }\end{array}$ & $\begin{array}{l}\text { Mar. Alg. } \\
\text { Tun. }\end{array}$ & $A C$ & Thérophyte & Cap de fer \\
\hline $\begin{array}{l}\text { Silene rosulata } \\
\text { S.W. \& Gr. } \\
\text { subsp. rosulata }\end{array}$ & & Caryophyllaceae & $\begin{array}{l}\text { End. (Quezel \& Santa, } \\
\text { 1962) }\end{array}$ & Mar. Alg. & $\mathrm{R}$ & Thérophyte & Cap de fer \\
\hline $\begin{array}{l}\text { Silene scabrida } \\
\text { S.W. \& Gr. }\end{array}$ & & Caryophyllaceae & $\begin{array}{l}\text { End. Num. (Quezel \& } \\
\text { Santa, 1962) } \\
\text { End.Alg.-Tun.(Pottier- } \\
\text { Alapetite, 1979) }\end{array}$ & Alg. Tun. & $\mathrm{R}$ & Thérophyte & Guerbes \\
\hline $\begin{array}{l}\text { Thymus } \\
\text { algeriensis Boiss. } \\
\text { \& Reut. }\end{array}$ & $\begin{array}{l}\text { T. ciliatus subsp. } \\
\text { algeriensis } \\
\text { (Boiss. \& Reut.) } \\
\text { Batt. } \\
\text { T. hirtus subsp. } \\
\text { algeriensis } \\
\text { (Boiss. \& Reut.) } \\
\text { Murb. }\end{array}$ & Lamiaceae & $\begin{array}{l}\text { End. N. A. (Quezel \& } \\
\text { Santa, 1963) } \\
\text { End. Mar.-Alg.-Tun.-Lib. } \\
\text { (El Oualidi et al., 2012) }\end{array}$ & $\begin{array}{l}\text { Mar. Alg. } \\
\text { Tun. Lib }\end{array}$ & $\mathrm{CC}$ & Chaméphyte & Chekaka \\
\hline \multicolumn{8}{|c|}{ Taxons non revus depuis plus de 50 ans } \\
\hline $\begin{array}{l}\text { Anthemis maritima } \\
\text { L. subsp. bolosii } \\
\text { Benedi \& Morelo }\end{array}$ & & Asteraceae & $\begin{array}{l}\text { Taxon non signalé par } \\
\text { Quezel \& Santa (1963) } \\
\text { End. Alg. (Dobignard \& } \\
\text { Chatelain, 2011a) }\end{array}$ & Alg. & $\begin{array}{l}\text { Non } \\
\text { signal } \\
\text { ée }\end{array}$ & $\begin{array}{l}\text { Hémicryptop } \\
\text { hyte }\end{array}$ & $\begin{array}{l}\text { Rochers } \\
\text { maritimes et } \\
\text { falaises de } \\
\text { Stora, rocher à } \\
\text { l'embouchure } \\
\text { de Saf Saf } \\
\text { (Oberprieler, } \\
\text { 1998) }\end{array}$ \\
\hline $\begin{array}{l}\text { Anthemis montana } \\
\text { Batt. non L. var. } \\
\text { punctata (Vahl.) } \\
\text { Batt. }\end{array}$ & A. punctata Vahl. & Asteraceae & $\begin{array}{l}\text { Taxon non signalé par } \\
\text { Quezel \& Santa (1963) } \\
\text { End.Alg-Tun. } \\
\text { (Oberprieler, 1998) }\end{array}$ & Alg.Tun. & $\begin{array}{l}\text { Non } \\
\text { signal } \\
\text { ée }\end{array}$ & $\begin{array}{l}\text { Hémicryptop } \\
\text { hyte }\end{array}$ & $\begin{array}{l}\text { Mont Gouffi } \\
\text { (Collo) } \\
\text { (Battandier \& } \\
\text { Trabut, 1888) }\end{array}$ \\
\hline $\begin{array}{l}\text { Anthemis } \\
\text { pedunculata Desf. } \\
\text { subsp. atlantica } \\
\text { (Pomel) Oberpr. }\end{array}$ & $\begin{array}{l}\text { A. atlantica } \\
\text { Pomel }\end{array}$ & Asteraceae & $\begin{array}{l}\text { Taxon non signalé par } \\
\text { Quezel \& Santa (1963) } \\
\text { End.Alg.-Tun. } \\
\text { (Oberprieler, 1998) }\end{array}$ & Alg.Tun. & $\begin{array}{l}\text { Non } \\
\text { signal } \\
\text { ée }\end{array}$ & $\begin{array}{l}\text { Hémicryptop } \\
\text { hyte }\end{array}$ & $\begin{array}{l}\text { Mont Gouffi } \\
\text { (Collo), mont } \\
\text { Filfilla } \\
\text { (Oberprieler, } \\
\text { 1998) }\end{array}$ \\
\hline
\end{tabular}




\begin{tabular}{|c|c|c|c|c|c|c|c|}
\hline $\begin{array}{l}\text { Armeria } \\
\text { choulettiana } \\
\text { Pomel }\end{array}$ & $\begin{array}{l}\text { A. plantaginea } \\
\text { subsp. } \\
\text { choulettiana } \\
\text { (Pomel) Maire } \\
\text { Statice } \\
\text { plantaginea } \\
\text { subsp. } \\
\text { choulettiana } \\
\text { (Pomel) Maire }\end{array}$ & Plumbaginaceae & $\begin{array}{l}\text { Non signalée par Quezel } \\
\text { \& Santa (1963) } \\
\text { End. Mar.-Alg.-Tun. (El } \\
\text { Oualidi et al., 2012) }\end{array}$ & Mar.Alg.Tun & AR & $\begin{array}{l}\text { Hémicryptop } \\
\text { hyte }\end{array}$ & $\begin{array}{l}\text { Ramdane } \\
\text { Djamel (ex } \\
\text { Saint Charles) } \\
\text { (P00083060; } \\
\text { Battandier \& } \\
\text { Trabut, 1888) }\end{array}$ \\
\hline $\begin{array}{l}\text { Euphorbia } \\
\text { cossoniana Boiss. }\end{array}$ & & Euphorbiaceae & $\begin{array}{l}\text { End. N.A. (Quezel \& } \\
\text { Santa, 1963) }\end{array}$ & $\begin{array}{l}\text { Mar. Alg. } \\
\text { Tun. }\end{array}$ & $\mathrm{R}$ & $\begin{array}{l}\text { Hémicryptop } \\
\text { hyte }\end{array}$ & $\begin{array}{l}\text { Skikda } \\
\text { (Battandier \& } \\
\text { Trabut, 1888) }\end{array}$ \\
\hline $\begin{array}{l}\text { *Lysimachia } \\
\text { cousiniana Coss. } \\
\text { \& Dur. }\end{array}$ & & Primulaceae & $\begin{array}{l}\text { End. (Quezel \& Santa, } \\
\text { 1963) } \\
\text { End Alg.-Tun (Pottier- } \\
\text { Alapetite, 1981) }\end{array}$ & Alg.Tun. & $A C$ & Thérophyte & 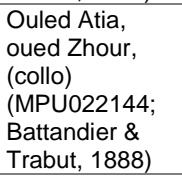 \\
\hline $\begin{array}{l}\text { *Moehringia } \\
\text { stellarioides Coss. }\end{array}$ & & Caryophyllaceae & $\begin{array}{l}\text { End. (Quezel \& Santa, } \\
\text { 1962) } \\
\text { End. Alg. (Dobignard \& } \\
\text { Chatelain, 2011b) }\end{array}$ & Alg. & $\mathrm{R}$ & $\begin{array}{l}\text { Hémicryptop } \\
\text { hyte }\end{array}$ & $\begin{array}{l}\text { Mont Gouffi à } \\
\text { Collo (MPU020 } \\
520 ; \\
\text { P05012843) }\end{array}$ \\
\hline $\begin{array}{l}\text { *Pedicularis } \\
\text { numidica Pomel }\end{array}$ & & Orobanchaceae & $\begin{array}{l}\text { End. (Quezel \& Santa, } \\
\text { 1963) } \\
\text { End. Alg. (Dobignard \& } \\
\text { Chatelain, 2013) }\end{array}$ & Alg. & $\mathrm{RR}$ & $\begin{array}{l}\text { Hémicryptop } \\
\text { hyte }\end{array}$ & $\begin{array}{l}\text { Aïn Sedma } \\
\text { (Collo) } \\
\text { (P00541524 ; } \\
\text { Battandier \& } \\
\text { Trabut, 1888) }\end{array}$ \\
\hline $\begin{array}{l}\text { Pinus pinaster } \\
\text { subsp renoui } \\
\text { (Villar) Maire }\end{array}$ & & Pinaceae & $\begin{array}{l}\text { Taxon non signalé par } \\
\text { Quezel \& Santa (1962) } \\
\text { End.Mar.- Alg. -Tun. } \\
\text { (IUCN, 2013) }\end{array}$ & Alg. Tun. & $\begin{array}{l}\text { Non } \\
\text { signal } \\
\text { ée }\end{array}$ & $\begin{array}{l}\text { Phanérophyt } \\
\text { e }\end{array}$ & $\begin{array}{l}\text { Sahel de Collo } \\
\text { (Maire, 1952) }\end{array}$ \\
\hline $\begin{array}{l}\text { *Satureja hispidula } \\
\text { (Boiss.\& Reut.) } \\
\text { Briq. }\end{array}$ & $\begin{array}{l}\text { Calamintha } \\
\text { hispidula Boiss. \& } \\
\text { Reut. } \\
\text { Clinopodium } \\
\text { hispidulum } \\
\text { (Boiss. \& Reut.) } \\
\text { Govaerts }\end{array}$ & Lamiaceae & $\begin{array}{l}\text { End. (Quezel \& Santa, } \\
\text { 1963) } \\
\text { End. Alg. (Dobignard \& } \\
\text { Chatelain, 2012) }\end{array}$ & Alg. & $\mathrm{RR}$ & Thérophyte & $\begin{array}{l}\text { Est de } \\
\text { Collo (Quezel \& } \\
\text { Santa, 1963) }\end{array}$ \\
\hline $\begin{array}{l}\text { *Sedum multiceps } \\
\text { Coss. \& Dur. }\end{array}$ & & Crassulaceae & $\begin{array}{l}\text { End. (Quezel \& Santa, } \\
\text { 1962) } \\
\text { End. Alg. (Dobignard } \\
\text { \&Chatelain, 2011b) }\end{array}$ & Alg. & $\mathrm{R}$ & Chaméphyte & $\begin{array}{l}\text { Collo (Battabdi } \\
\text { er \& Trabut, } \\
1888 \text { ) }\end{array}$ \\
\hline $\begin{array}{l}\text { *Silene colorata } \\
\text { Poir. } \\
\text { subsp. amphorina } \\
\text { (Pomel) Batt. }\end{array}$ & $\begin{array}{l}\text { S. amphorina } \\
\text { Pomel }\end{array}$ & Caryophyllaceae & $\begin{array}{l}\text { End. (Quezel \& Santa, } \\
\text { 1962) } \\
\text { End. Alg. (Dobignard \& } \\
\text { Chatelain, 2011b) }\end{array}$ & Alg. & $\mathrm{RR}$ & Thérophyte & $\begin{array}{l}\text { Oued Cherilla } \\
\text { au pied du } \\
\text { mont Filfilla } \\
\text { (MPU005821 ; } \\
\text { Battandier \& } \\
\text { Trabut, 1888) }\end{array}$ \\
\hline $\begin{array}{l}\text { Silene choulettii } \\
\text { Coss. }\end{array}$ & & Caryophyllaceae & $\begin{array}{l}\text { End. (Quezel \& Santa, } \\
\text { 1962) } \\
\text { End. Alg. (Dobignard \& } \\
\text { Chatelain, 2011b) }\end{array}$ & Alg. & $A C$ & Thérophyte & $\begin{array}{l}\text { Collo } \\
\text { (Battandier \& } \\
\text { Trabut, 1888) }\end{array}$ \\
\hline $\begin{array}{l}\text { Thymelaea } \\
\text { microphylla Coss. } \\
\text { \& Dur. }\end{array}$ & & Thymelaeaceae & $\begin{array}{l}\text { End. N.A. (Quezel \& } \\
\text { Santa, 1963) } \\
\text { End. Mar.- Alg.- Tun.- Lib. } \\
\text { (El Oualidi et al., 2012) }\end{array}$ & $\begin{array}{l}\text { Mar. } \\
\text { Alg.Tun. } \\
\text { Lib. }\end{array}$ & $\mathrm{CC}$ & Chaméphyte & $\begin{array}{l}\text { Garaet Sidi } \\
\text { Makhlouf } \\
\text { (herbier ENSA) }\end{array}$ \\
\hline
\end{tabular}

\section{Discussion}

La flore endémique de la wilaya de Skikda représente plus d'un $1 / 8$ de la flore endémique de l'Algérie du nord qui compte 407 taxons (Véla \& Benhouhou, 2007).

Les familles retrouvées dominantes dans notre région sont considérées comme les plus riches en plantes endémiques à l'échelle nationale; les Asteraceae, les Caryophyllaceae et les Fabaceae occupent les trois premières positions (Quezel, 1964).

Le genre Genista avec ses 5 taxons endémiques dont deux endémiques algériennes ( $G$. numidica subsp numidica et $G$. vepres) est de loin le mieux représenté dans la région. A l'échelle nationale, il compte parmi les genres les plus riches en taxons endémiques (Quezel, 1964) avec 11 taxons répartis sur tout le pays (Quezel \& Santa, 1961). Au niveau régional, toutes les espèces et sous-espèces du genre sont signalées dans, au moins, deux localités, à l'exception du G. vepres. Les nombreux pieds que nous avons observés sur le mont Gouffi à $1000 \mathrm{~m}$ d'altitude, vivaient en association avec $G$. tricuspidata et Quercus x numidica.

Le genre Silene, représenté chez nous par 4 taxons, est classé en tête de liste au niveau national avec 18 taxons endémiques (Quezel, 1964). C'est au sein de ce genre que nous trouvons Silene colorata subsp amphorina signalée pour toute l'Algérie uniquement à Skikda (Battandier \& Trabut, 1888 ; Maire, 1963). 
Au niveau de la wilaya, la distribution de Quercus afares, $Q$. canariensis et $Q$. x numidica se limite au massif de Collo. Après plus d'un demi-siècle de leur signalisation dans la flore de l'Afrique du Nord (Maire, 1961), ces taxons forestiers existent encore aujourd'hui, leur préservation est probablement due à l'inaccessibilité des sites de prolifération. Les montagnes constituent, en effet, des sites de refuges et des points de préservation importants pour plusieurs espèces (Médail \& Diameda, 2009).

Les endémiques algériennes strictes de la wilaya représentent $8 \%$ des endémiques algériennes strictes de l'Algérie du nord qui comptent 224 taxons (Véla \& Benhouhou, 2007). Les taxons à rechercher, ayant ce statut, ont été majoritairement signalées dans la région de Collo. Cela reflète, d'un côté, la richesse floristique exceptionnelle de la région, renforçant sa classification comme une zone importante pour les plantes (ZIP) (Yahi et al., 2012) et, d'un autre côté, le besoin d'intensifier les inventaires floristiques et les études botaniques dans cette zone qui demeure jusqu'à présent très mal explorée. Les endémiques algéro-tunisiennes de la wilaya représentent $51,72 \%$ des endémiques algéro-tunisiènnes de l'Algérie du nord qui comptent 58 taxons (Véla \& Benhouhou, 2007). Cette prédominance des endémiques partagées avec la Tunisie ne suscite pas l'étonnement vu la proximité de la wilaya de la frontière tunisienne, par contre, elle est très loin de la frontière marocaine ce qui fait des endémiques algéro-marocaines les moins abondantes dans la région.

Le changement de la chorologie de plusieurs taxons peut être expliqué par l'accroissement des investigations, que ce soit en méditerranée ou en Afrique du nord, visant à inventorier la biodiversité et à suivre l'évolution de cette dernière en fonction de la dynamique naturelle des espèces végétales permettant d'élargir les listes floristiques par l'ajout de taxons ou de les rétrécir par le retrait de d'autres. $\mathrm{Ce}$ changement met également le point sur l'importance des inventaires dans la détermination du statut biogéographique des taxons.

La Numidie littorale arrive en tête en ce qui concerne les espèces rares (Véla \& Benhouhou, 2007). La flore endémique assez rare, rare ou très rare est considérée comme vulnérable, marquant ainsi la grande importance de la mise en œuvre des projets de conservation.

La prolifération de l'Acacia saligna à Filfilla est particulièrement préoccupante. L'espèce avance de plus en plus vers les terrains internes mettant ainsi en danger toute la richesse biologique signalée dans la région. Les incendies déclenchés en mois d'Août 2017, nous ont permis de découvrir l'ampleur de la progression de l'A. saligna dans le maquis arboré, des centaines de jeunes troncs carbonisés sont facilement reconnaissables grâce à leur silhouette caractéristique. D'après Jeanmonod (1998) la pénétration d'une espèce allochtone dans des milieux naturels est un indice de menace pour la flore indigène. A Ben Azouz et à Guerbes le caractère invasif de cette plante australienne est clairement observable. Elle s'installe parmi le Myrtus communis et l'Olea europaea, en populations très denses montrant une dynamique d'extension rapide. Cette espèce est responsable de la diminution de la richesse spécifique, de la diversité et de l'abondance de la flore autochtone (Abd El-Gawad \& El-Amier, 2015), de l'altération de la composition floristique (Del Vicchio et al., 2013) et du changement dans le sol, de la structure, la diversité et la fonction des communautés microbiennes (Crisóstomo et al., 2013). Malgré son action néfaste sur l'environnement, l'A. saligna est encore utilisée dans les programmes de reboisement comme elle figure sur les répertoires de vente des pépinières locales (Sakhraoui et al., 2019). Une action d'arrachage devrait être entreprise au plus vite au niveau de ces localités pour pouvoir garder toute l'authenticité floristique qui les caractérise; rappelons que dans la liste actualisée, 5 taxons endémiques ont été enregistrés à Filfilla et 24 à Guerbes-Sanhadja, à ceux-là s'ajoute 3 taxons observés à Filfilla, signalés dans la liste à rechercher parmi lesquels nous trouvons Silene colorata subsp amphorina. Cette opération devrait s'accompagner d'une interdiction de la vente et de la culture de l' $A$. saligna car l'arrachage n'est pas efficace si l'espèce visée est encore cultivée et/ou vendue (Jeanmonod, 1998).

L'Arundo donax est une autre espèce problématique dans la wilaya. Elle domine le couvert végétal aux bords des cours d'eau et y est souvent associée à l'Ailanthus altissima. La végétation ripisylve disparait totalement dans certains endroits pour laisser place à ces deux plantes envahissantes.

Le Carpobrotus edulis est majoritairement présente sur les dunes littorales de la partie Est de la ville de Skikda où elle couvre de grandes étendues notamment sur les plages de la plaine de GuerbesSanhadja. La flore endémique recensée dans ces endroits fragiles (8 taxons endémiques de la liste actualisée ont été observés au niveau des plages) est sérieusement mise en danger par la prolifération de populations denses et serrées de cette espèce.

La naturalisation en elle-même de l'Opuntia ficusindica ne constitue pas, du moins dans l'immédiat, un danger pour la flore indigène, c'est plutôt le grand intérêt que porte la population rurale et les institutions étatiques pour la culture de cette cactacée qui pourrait constituer une véritable menace pour les milieux naturels de la région. Bien qu'elle soit très estimée, l'O. ficus-indica est responsable de plusieurs effets néfastes, compétition avec la végétation autochtone et expulsion de cette dernière (Weber, 2017), son utilisation dans les projets de réhabilitation devrait se limiter aux zones arides et semi-arides du pays.

La prolifération de toutes ces espèces, déclarées envahissantes en méditerranée, dans les milieux naturels de la wilaya ne fait que compliquer l'opération de conservation d'autant plus que ces milieux sont déjà sous l'effet de plusieurs menaces notamment le défrichement, les incendies et l'exploitation agricole (Toubal et al., 2014).

Vu l'importance incontestée de la flore endémique, un projet de conservation ex situ est en cours de 
réalisation, il s'agit de la création d'un jardin spécifique à cette catégorie de plantes au sein des jardins de l'Université de Skikda.

En se référant au décret exécutif $n^{\circ} 12-03$ fixant la liste des espèces végétales non cultivées protégées à l'échelle nationale (JORA, 2012), 15 taxons seulement, figurant dans les listes établies dans ce travail, jouissent d'un statut de protection (Tab. 1, 2), représentant à peine $23 \%$ du potentiel floristique endémique recensé dans la wilaya. Des efforts plus déterminés devraient être déployés pour parvenir à sauvegarder toute la biodiversité décrite dans les différents milieux du pays à commencer par la révision de la liste des espèces protégées.

Bien qu'il existe en Algérie d'innombrables travaux sur la flore endémique, les données demeurent parcellaires. Cette situation, nous a empêché de réaliser des comparaisons avec les autres wilayas du pays par rapport à la richesse floristique globale. Néanmoins, les taxons endémiques partagés avec les autres régions limitrophes peuvent être déterminés. C'est ainsi que Skikda partage avec Jijel: Carduus numidicus, Pinus pinaster subsp renoui, Silene rosulata subsp rosulata et Urginea maritima var. numidica, (Bouldjedri, 2013; Khennouf et al., 2018), alors qu'elle partage avec Annaba un nombre de taxons plus important: Brassica fruticosola subsp. numidica, Carduus numidicus, Cyclamen africanum, Galactites mutabilis, Genista ferox, G. numidica, G. ulicina, Linum numidicum, Lotus drepanocarpus, Mathiola sinuata subsp numidica, Scilla lingulata, $S$. numidica, Scrophularia tenuipes et Silene rosulata subsp. rosulata (Hamel et al., 2013; Boulemtafes et al., 2018).

Tableau 3. Les espèces naturalisées dans les zones prospectées, nature des milieux colonisés et moyen de propagation.

Table 3. Species naturalized in the prospected areas, nature of colonized environments and means of propagation.

\begin{tabular}{|c|c|c|c|}
\hline Espèces & Lieu d'observation & Milieu colonisé & $\begin{array}{l}\text { Moyen de propagation dans } \\
\text { la zone d'étude }\end{array}$ \\
\hline Acacia saligna & $\begin{array}{l}\text { Filifilla } \\
\text { Ben Azouz } \\
\text { Djendel } \\
\text { Skikda }\end{array}$ & $\begin{array}{l}\text { - Maquis arboré } \\
\text { - Maquis clairs } \\
\text { - Bords des routes }\end{array}$ & $\begin{array}{l}\text { Graines et drageons souvent } \\
\text { facilitée par l'homme }\end{array}$ \\
\hline Ailanthus altissima & Toutes les communes & $\begin{array}{l}\text { - Bords des routes } \\
\text { - Bords des oueds } \\
\text { - Terrains de construction } \\
\text { abandonnés }\end{array}$ & Graines et drageons \\
\hline Arundo donax & Toutes les communes & $\begin{array}{l}\text { - Bords des oueds } \\
\text { - Bords des champs } \\
\text { - Bords des routes }\end{array}$ & $\begin{array}{l}\text { Végétatives, par les rhizomes } \\
\text { souvent facilitée par l'homme }\end{array}$ \\
\hline $\begin{array}{l}\text { Austrocylindropuntia } \\
\text { subulata }\end{array}$ & Ben Azouz & $\begin{array}{l}\text { - Bords des routes } \\
\text { - Autour des habitations } \\
\text { rurales }\end{array}$ & $\begin{array}{l}\text { Végétative, facilitée par } \\
\text { l'homme }\end{array}$ \\
\hline Carpobrotus edulis & $\begin{array}{l}\text { Djendel } \\
\text { Filfilla } \\
\text { Skikda } \\
\text { Filfilla }\end{array}$ & $\begin{array}{l}\text { - Dunes littorales } \\
\text { - Plages } \\
\text { - Talus }\end{array}$ & $\begin{array}{l}\text { Essentiellement par les } \\
\text { stolons, facilitée par l'homme }\end{array}$ \\
\hline Nicotiana glauca & $\begin{array}{l}\text { Hamadi Krouma } \\
\text { Skikda }\end{array}$ & -Bords des routes & Graines \\
\hline $\begin{array}{l}\text { Opuntia ficus-indica } \\
\text { var. amyclaea } \\
\text { var. ficus-indica }\end{array}$ & Toutes les communes & $\begin{array}{l}\text { - Maquis clairs } \\
\text { - Falaises } \\
\text { - Bords des champs } \\
\text { - Autour des habitations } \\
\text { rurales }\end{array}$ & $\begin{array}{l}\text { Végétative, par les raquettes, } \\
\text { souvent facilitée par l'homme }\end{array}$ \\
\hline Opuntia stricta & $\begin{array}{l}\text { Filfilla } \\
\text { Skikda }\end{array}$ & $\begin{array}{l}\text { - Terrains incultes } \\
\text { - Entre les habitations } \\
\text {-Bord des routes } \\
\text {-Bords des routes }\end{array}$ & $\begin{array}{l}\text { Végétative, par les raquettes } \\
\text { quelques fois facilitée par } \\
\text { l'homme }\end{array}$ \\
\hline Oxalis pes-caprae & Toutes les communes & $\begin{array}{l}\text {-Vergers d'agrumes } \\
\text {-Contour des champs } \\
\text { - Décombres }\end{array}$ & Bulbes et bulbilles \\
\hline Ricinus communis & Toutes les communes & $\begin{array}{l}\text { - Bords des oueds } \\
\text { - Bords des routes } \\
\text { - Ravins }\end{array}$ & Graines \\
\hline Tropaeolum majus & $\begin{array}{l}\text { Filfilla } \\
\text { Skikda }\end{array}$ & $\begin{array}{l}\text {-Voisinage des jardins } \\
\text {-Décombres }\end{array}$ & Graines \\
\hline
\end{tabular}

\section{Conclusion}

La flore indigène de la wilaya de Skikda est très riche en plantes endémiques. La liste élaborée dans ce travail, comprenant 30 espèces, 6 sous-espèces et
2 variétés, risque de s'élargir si les taxons à rechercher sont retrouvés sur son territoire. Les futures investigations floristiques devront se focaliser sur la recherche de ces taxons pour rendre possible leur préservation. 
Les zones importantes pour les plantes endémiques sont Guerbes-Sanhadja, Cap de Fer, Filfilla, Stora et Collo.

La flore endémique régionale est loin d'être suffisamment connue. Un large périmètre de la wilaya demeure encore très mal exploré. Toute la zone située à l'ouest de la ville de Skikda, notamment la zone côtière des falaises maritimes et les zones plus internes, situées essentiellement dans le massif de Collo, mérite d'être profondément prospectée.

Les espèces envahissantes ou à potentiel envahissant constituent une menace émergente pour cette biodiversité d'intérêt majeur. L'Ailanthus altissima et Arundo donax sont majoritairement menaçantes pour la végétation ripisylve. Le Carpobrotus edulis est menaçant pour la végétation des dunes littorales, alors que, l'Acacia saligna et Opuntia ficus-indica sont menaçantes pour la végétation des maquis clairs et arborés, leur culture ne devrait plus être pratiquée et leurs populations développées dans les milieux naturels notamment à Guerbes-Sanhadja et Filfilla devraient être éradiquées.

\section{Bibliographie}

Abd El-Gawad, A.M. \& El-Amier, Y.A. (2015). Allelopathy and Potential Impact of Invasive Acacia saligna (Labill.) Wendl., on Plant Diversity in the Nile Delta Coast of Egypt. International Journal of Environmental Research, 9(3), 923-932.

doi: https://doi.org/10.22059/ijer.2015.979

Abdi, S. (2015). Structure et écologie des canards plongeurs (Anatidés) dans les zones humides de Guerbes-Sanhadja (wilaya de Skikda, Nordest de l'Algérie). Thèse de Doctorat $3{ }^{\text {ème }}$ cycle : Université Mohamed Chérif Messaadia, Souk Ahras (Algérie).

APG IV. (2016). An update of the Angiosperm Phylogeny Group classification for the orders and families of flowering plants: APG IV. Botanical Journal of The Linnean Society, 181 (1): 1-20. doi: https://doi.org/10.1111/boj.12385

Battandier, J.A. \& Trabut, L.C. (1888). La flore de l'Algérie. Dicotylédones. Alger, Paris.

Bouldjedri, M. (2013). Ecologie d'un hydrosystème de la région de Jijel: Cas de la zone humide de Beni-Belaid. Thèse de doctorat: Université Badji Mokhtar, Annaba (Algérie).

Boulemtafes, A., Hamel, T., de Bélair G. \& Véla, E. (2018). Nouvelles données sur la distribution et l'écologie de seize taxons végétaux du littoral de la péninsule de l'Edough (Nord-Est algérien). Bulletin de la. Société Linnéenne de Provence, 69, 1-18.

Brunel, S. \& Tison, J. M. (2005). A method of selection and hierarchization of the invasive and potentially invasive plants in continental Mediterranean France. In: Brunel, S. (eds.), Proceedings of the International Workshop: Invasive Plants in Mediterranean Type Regions of the World (pp. 27-36). Mèze, France: Council of Europe publishing.

Capdevilla Argüelles, L., Iglesias Garcia, A., Orueta, J.F. \& Zilleti, B. (2006). Especies exoticas invasoras: Dignostico y bases para la prevencion y el manejo. Série Técnica. Madrid: Naturaleza y Parques Nationales.

Celesti-Grapow, I., Alessandrini, A., Arrigoni, P.V., Banfi, E., Bernardo, I., Bovio, M., Brundu, G., Cagiotti, M. R., Camarda, I., Carli, E., Conti, F., Fascetti, S., Galasso, G., Gubellini, I., La Valva, V., Lucchese, F., Marchiori, S., Mazzola, P., Peccenini, S., Poldini, I., Pretto, F., Prosser, F., Siniscalco, C., Villani, M. C., Viegi, I., Wilhalm, I. \& Blasi, C. (2009). Inventory of the non-native flora of Italy. Plant Biosystems, 143, 386-430.

doi: https://doi.org/10.1080/11263500902722824

Crisóstomo, J. A., Rodriguez-Echeverria, S. \& Freitas, H. (2013). Co-introduction of exotic rhizobia to the rhizosphere of the invasive legume Acacia saligna, an intercontinental study. Applied Soil Ecology, 64, 118-126. doi: https://doi.org/10.1016/j.apsoil.2012.10.005

De Belair, G. \& Sameraoui B. (2000). L'écocomplexe des zones humides de Beni-Belaid un projet de réserve naturelle. Sciences \& Technologie, 114-124.

Del Vecchio, S., Acosta, A. \& Stanisci, A. (2013). The impact of Acacia saligna invasion on Italian coastal dune EC habitats. Comptes Rendus Biologies, 336, 364-369. doi: http://dx.doi.org/10.1016/j.crvi.2013.06.004

Dobignard, A. \& Chatelain, C. (2010-2011a2011 b-2012 et 2013). Index synonymique de la flore d'Afrique du Nord. 5 Vol. Genève: Conservatoire et Jardin botaniques de la Ville de Genève.

El Oualidi, J., Khamar, H., Fennane, M., Ibn Tattou, M., Chauvet, S. \& Taleb, M.S. (2012). Checkliste des endémiques et spécimens types de la flore vasculaire de l'Afrique du Nord. Document de l'institut scientifique, $\mathrm{n}^{\circ} 25$, Université Mohammed V-Agdal, Rabat, Maroc.

Emberger, L. (1955). Une classification biogéographique des climats. Recueil des Travaux des Laboratoires de Botanique et de Géologie de la Faculté des. Sciences, 7, 3-43.

Fennane, M. \& Ibn Tattou, M. (1998). Catalogue des plantes vasculaires, rares, menacées ou endémiques du Maroc. Bocconea, 8, 115-233.

Hamel, T. \& Meddad-Hamza, A. (2016). Note sur les Orchidées de la péninsule de l'Edougn (Nord-est algérien). L'Orchidophile, 211, 367- 374 .

Hamel, T., Meddad-Hamza, A. \& Mabarek Oudina, A. (2017a). De nouvelles perspectives pour les orchidées de la région de Skikda (Nord-Est algérien). Journal Europäischer Orchideen, 49(1), 61-78.

Hamel, T., Slimani, A. \& Seridi, R. (2017b). Flora Diversity of Garaet Ouajaa (Wetlands of Guerbes-Senhadja, Northeast Algeria. 
American Journal of Life Science Researches, 5 (3), 102-109.

Hamel, T., Seridi, R., De Belair, G., Slimani, A. \& Babali, B. (2013). Flore vasculaire rare et endémique de la péninsule de l'Edough (NordEst Algérien). Revue des Sciences et de la.Technologie, Synthèse, 26, 65-74.

IUCN. (2013). International Union for Conservation of Nature, Red List of Threatened Species (ver. 2013.1). Available at: http://www.iucnredlist.org, (22/2/2019).

Jeanmonod, D. (1998). Les plantes introduites en Corse : impacts, menaces et propositions de protection de la flore insulaire. Biocosme Mésogéen, 15 (1), 45-68.

JORA. (2012). Journal Officiel de la République Algérienne Démocratique et Populaire, $n^{\circ} 3$.

Khennouf, H., Chefrour, A., Corcket, E., Alard, D. \& Véla, E. (2018). La végétation dunaire du littoral de Jijel (Algérie): Proposition d'une nouvelle zone importante pour les plantes. Revue d'Ecologie (Terre et Vie), 73 (3), 344361.

Le Floc'h, E., Boulos, L. \& Véla, E. (2010). Catalogue synonymique commenté de la flore de Tunisie. Tunis: Ministère de l'environnement et du développement durable, banque nationale de gènes.

Maire, R. (1952-1958-1961-1963 et 1987). Flore de l'Afrique du Nord, Pteridophyta, Gymnospermae, Monocotyledonae. Dicotyledonae. Paris: Paul Lechevalier.

Médail, F. \& Diadema, K. (2009). Glacial refugia influence plant diversity patterns in the Mediterranean Basin. Journal of Biogeography, 1-13.

doi:https://doi.org/10.1111/j.13652699.2008.02 051.x

Meghzili, H. (2015). Modèles d'aménagement et d'urbanisation des zones d'expansion touristique de la wilaya de Skikda (Algérie). Thèse de doctorat: Université de Bretagne Occidentale, Brest (France).

Oberprieler, C. (1998). The Systematics of Anthemis L. (Compositae, Anthemideae) in W and C North Africa. Bocconea, 9, 1-328.

Pottier-Alapetite, M. (1979-1981). Flore de la Tunisie, Angiospermes-Dicotylédones, (Apétales-Dialypétales, Gamopétales). Parties 1 et 2. Tunis: Ministre de l'Enseignement Supérieur et de la Recherche Scientifique et le Ministère de l'Agriculture.

Quezel, P. (1964). L'endémisme dans la flore de l'Algérie. Comptes rendus des séances de la Société de Biogéographie, 361, 137- 149.
Quezel, P. \& Santa, S. (1962-1963). Nouvelle flore de l'Algérie et des régions désertiques méridionales. Tome 1 et 2. Paris: Centre nationale de la recherche scientifique.

Raunkiaer, C. (1934). The life forms of plants and statistical plant, Geography. Oxford: Claredon press.

Sakhraoui, N., Metallaoui, S., Chefrour, A. \& Hadef, A. (2019). La flore exotique potentiellement envahissante d'Algérie: première description des espèces cultivées en pépinières et dans les jardins. Biotechnologie, Agronomie, Société et Environnement, 23 (2), 63-73. doi: https://doi.org/10.25518/17804507.17902

Thiers, B. [continuously updated]. Index Herbariorum: A global directory of public herbaria and associated staff. New York Botanical Garden's Virtual Herbarium, http://sweetgum.nybg.org/science/ih/, (21/2/2019).

Toubal, O., Boussehaba, A., Toubal, A. \& Samraoui, B. (2014). Biodiversité méditerranéenne et changements globaux: cas du complexe de zones humides de Guebes-Senhadja. Physio-Géo, Géographie Physique et Environnement, 8, 273-295.

Véla, E. (2018). De l'inventaire de la biodiversité aux propriétés de conservation dans le hotspot du bassin méditerranéen : peut-on combler les déficits de connaissance? Mémoire d'habilitation à diriger des recherches: Université de Montpellier (France).

Véla, E. \& Benhouhou, S. (2007). Evaluation d'un nouveau point chaud de biodiversité végétale dans le bassin méditerranéen (Afrique du Nord). Comptes Rendus Biologies, 330, 589605. doi: https://doi.org/10.1016/j.crvi.2007.04.006

Véla, E., De Belair, G., Rosato, M. \& Rosselo, J. (2016). Taxonomic remarks on Scilla anthericoides Poir. (Asparagaceae, Scilloideae) a neglected species from Algeria. $\begin{array}{lll}\text { Phytotaxa, } 288 & \text { (2), } 154-160 . & \text { doi: }\end{array}$ http://dx.doi.org/10.11646/phytotaxa.288.2.5

Weber, E. (2017). Invasive plant species of the world: $A$ reference guide to environmental weeds (2 ed.). Wallingford, Oxfordshine, Boston, MA: CABI Publishing.

Yahi, N., Véla, E., Benhouhou, S., De Belair, G. \& Gharzouli R. (2012). Identifying Important Plants Areas (Key Biodiversity Areas for Plants) in northern Algeria. Journal of Threatened Taxa, 4 (8), 2753-2765. doi: https://doi.org/10.11609/JoTT.o2998.2753-65. 